\title{
Clase social, desigualdades en salud y conductas relacionadas con la salud de la población trabajadora en Chile
}

\author{
Kátia Bones Rocha, ${ }^{1}$ Carles Muntaner, ${ }^{2}$ María José González Rodríguez, ${ }^{3}$ \\ Pamela Bernales Baksai, ${ }^{3}$ Clelia Vallebuona, ${ }^{4}$ Carme Borrell ${ }^{5}$ y Orielle Solar ${ }^{3}$
}

Forma de citar Rocha KB, Muntaner C, González Rodríguez MJ, Bernales Baksai P, Vallebuona C, Borrell C, et al. Clase social, desigualdades en salud y conductas relacionadas con la salud de la población trabajadora en Chile. Rev Panam Salud Publica. 2013;33(5):340-8.

RESUMEN Objetivo. Analizar los vínculos entre la clase social y los diferentes indicadores y conductas relacionados con la salud, a partir de una perspectiva neomarxista en población trabajadora chilena.

Métodos. Se realizó un estudio transversal a partir de la Primera Encuesta Nacional de Condiciones de Empleo, Trabajo, Salud y Calidad de Vida de los Trabajadores y Trabajadoras en Chile, efectuada en 2009-2010 ( $\mathrm{n}=9$ 503). Las variables dependientes fueron el estado de salud autopercibido y la salud mental, examinada mediante el Cuestionario de Salud Global (GHQ-12, por sus siglas en inglés). Las variables de conductas relacionadas con la salud incluyeron el consumo de tabaco y la realización de actividad física. La variable independiente fue la clase social neomarxista. Se realizaron análisis descriptivos de prevalencia y se estimaron modelos de razón de probabilidades (RP) e intervalos de confianza de 95\% (IC95\%).

Resultados. Los medianos empresarios refirieron tener una menor prevalencia de mala salud (21,6\% [RP 0,68; IC95\% 0,46-0,99]). En relación a la salud mental, los que presentaban menor riesgo eran los gerentes básicos (RP 0,43; IC95\% 0,21-0,88), observándose diferencias entre hombres y mujeres. Los que refirieron fumar con menor frecuencia fueron los empresarios, mientras que los que realizaban significativamente más actividad física fueron los empresarios, los supervisores expertos y los trabajadores semicalificados.

Conclusiones. Los empresarios y gerentes expertos son los que presentan mejores indicadores y conductas relacionados con la salud. El proletario formal, el proletario informal y los supervisores básicos, en cambio, son los que presentan los peores indicadores de salud global, confirmando así que la clase social es un determinante clave en la generación de desigualdades en materia de salud de la población.

Palabras clave Clase social; desigualdades en la salud; tabaquismo; actividad motora; género y salud; conductas saludables; Chile.

La perspectiva de clase social neomarxista (CSNM) constituye una valiosa al-

Pontifícia Universidade Católica do Rio Grande do Sul (PUCRS), Porto Alegre, Brasil. La correspondencia se debe dirigir a Kátia Bones Rocha. Correo electrónico: katiabonesrocha@gmail.com

2 University of Toronto, Toronto, Canadá.

Universidad Mayor, Santiago, Chile.

Ministerio de Salud de Chile, Santiago, Chile.

5 Agència de Salut Pública de Barcelona (ASPB), Barcelona, España. ternativa frente a la estratificación social, al poner el foco en las relaciones de propiedad y control sobre los recursos productivos -físicos, financieros y organizativos- como principal determinante de las desigualdades sociales - y de salud (1). Contrariamente a los análisis que utilizan el concepto de estratificación social, centrados en categorías nominales $\mathrm{u}$ ordinales y jerarquías - como nivel de estudios, ingresos u ocupación-, la CSNM parte de comprender la forma en que las sociedades capitalistas son sistemáticamente estructuradas a partir de la división de clases y cómo los actores sociales se vinculan a través de relaciones de producción, propiedad, dominación y control sobre la toma de decisiones 
en el trabajo (2). La ventaja de utilizar indicadores y realizar mediciones sobre aspectos sociales — como propone el modelo de CSNM - es la posibilidad de adentrarse con mayor profundidad en las verdaderas causas de las desigualdades observadas en el campo de la salud.

La investigación en epidemiología social demuestra con claridad el impacto que tiene la clase social en la salud de la población, incluso después de haber considerado los efectos de los indicadores tradicionales como educación, ingresos y ocupación (3-8). La CSNM, a diferencia de los enfoques convencionales, despliega un abanico de nuevos instrumentos - como la posición contradictoria de clase- que permiten arribar a otro tipo de resultados (1-2) La posición contradictoria de clase, por ejemplo, ha probado que los supervisores tienen una peor salud mental que sus supervisados, quienes se encuentran por debajo en la jerarquía de poder y económica de la empresa. Esto se explica por el estrés que se origina por su ubicación jerárquica, entre los gerentes -mayor control y poder de decisión en la empresa- y la clase trabajadora (1-2). Sin embargo, y a pesar de la valiosa información que aporta este tipo de enfoques, los indicadores de estratificación social dominan la literatura epidemiológica actual, en gran parte debido a consideraciones pragmáticas, como una mayor disponibilidad de datos o la simplicidad intuitiva de las jerarquías tradicionales $(9,10)$.

Con respecto a la salud mental, las mediciones de CSNM son especialmente útiles para entender los mecanismos que pueden coadyuvar para que los trabajadores presenten alguna de estas afecciones, en función de las relaciones de producción de su ambiente laboral (5-6). Varios estudios han comprobado la asociación entre clase social y variables como salud autopercibida, salud mental y conductas relacionadas con la salud $(3,6-7)$. El tabaquismo, por ejemplo, empieza primero en hombres y en personas de clases privilegiadas, para después pasar a las mujeres y personas de clases trabajadoras (11). Sobre la actividad física, otra importante variable de salud pública, hay estudios que muestran que las personas de clases trabajadoras realizan menos ejercicio físico $(12,13)$, mientras que otros notifican una mayor prevalencia de inactividad en las clases sociales más privilegiadas (14).
Un trabajo de revisión de literatura científica, realizado en América Latina, destaca la escasez de análisis empíricos que traten la relación entre clase social y salud, con base en los marcos teóricos neomarxistas y neoweberianos (15), a diferencia de lo que ocurre en Europa y Estados Unidos, donde existe una larga tradición en este tipo de investigaciones sociales (16).

En este sentido, la Primera Encuesta Nacional de Condiciones de Empleo, Trabajo, Salud y Calidad de Vida de los Trabajadores y Trabajadoras en Chile (ENETS, 2009-2010) ha permitido conocer la distribución por clase social de la población chilena, así como analizar la asociación entre los indicadores y conductas relacionados con la salud y la clase social, desde una perspectiva neomarxista basada en los trabajos de Wright (1). Además, cabe destacar que en la encuesta - y, por ende, en el análisis- se ha estratificado según el género, lo que reviste particular importancia toda vez que la clase social puede tener diferente impacto en la salud de hombres y mujeres (17).

El presente estudio se propone precisamente analizar los vínculos entre la clase social y los diferentes indicadores y conductas relacionados con la salud, a partir de una perspectiva neomarxista en población trabajadora chilena.

\section{MATERIALES Y MÉTODOS}

\section{Diseño, fuente y población de estudio}

Se realizó un estudio de diseño transversal utilizando los datos de la Primera Encuesta Nacional de Condiciones de Empleo, Trabajo, Salud y Calidad de Vida de los Trabajadores y Trabajadoras en Chile (2009-2010). El diseño de la muestra fue probabilístico, por estratos geográficos y representativo de zonas urbanas y rurales (18).

La población de estudio incluyó a residentes urbanos y rurales de 15 o más años de edad que al momento de la encuesta tuviesen trabajo o, en caso de estar desocupados, que hubiesen trabajado durante los 12 meses anteriores a la encuesta $(n=9503)$. Las entrevistas fueron realizadas en el hogar de la persona encuestada, por personal capacitado, en el período de septiembre de 2009 a octubre de 2010.

\section{Variables de estudio}

\section{Clase social neomarxista}

En este estudio se utiliza un esquema de clase social basado en el modelo de Wright (1), con adaptaciones (5). Dicho esquema contiene 12 categorías -o posiciones de clase- distribuidas en tres dimensiones, a saber: (i) relaciones de propiedad, (ii) control sobre la organización del trabajo y (iii) credenciales/habilidades (figura 1). Cada una de estas 12 posiciones fue obtenida a partir de un conjunto de preguntas incluidas en el cuestionario (véase material suplementario). Asimismo, se diferenció entre proletario formal — con contrato- e informal — sin contrato-, lo que es un punto novedoso puesto que por primera vez se incluye esa distinción en una clasificación de CSNM. Tal inclusión se ve justificada porque gran parte de la población de América Latina trabaja sin un contrato formal (19).

\section{Variables dependientes}

Salud

Estado de salud autopercibido. Se construyó a partir de la pregunta "En general, us-

\section{FIGURA 1. Esquema de clase social neomarxista de Erik Wright}

\begin{tabular}{|c|c|c|c|c|}
\hline & Propietarios & & Asalariadc & \\
\hline & $\begin{array}{l}\text { 1. Empresarios } \\
\text { (más de } 10 \text { empleados) }\end{array}$ & $\begin{array}{l}\text { 4. Gerentes } \\
\text { expertos }\end{array}$ & $\begin{array}{l}\text { 7. Gerentes } \\
\text { semicalificados }\end{array}$ & $\begin{array}{l}\text { 10. Gerentes } \\
\text { básicos }\end{array}$ \\
\hline $\begin{array}{c}\text { Bienes de } \\
\text { organización }\end{array}$ & $\begin{array}{l}\text { 2. Medianos empresarios } \\
\text { (entre } 2 \text { y } 10 \text { empleados) }\end{array}$ & $\begin{array}{l}\text { 5. Supervisores } \\
\text { expertos }\end{array}$ & $\begin{array}{l}\text { 8. Supervisores } \\
\text { semicalificados }\end{array}$ & $\begin{array}{l}\text { 11. Supervisores } \\
\text { básicos }\end{array}$ \\
\hline & $\begin{array}{l}\text { 3. Pequeña burguesíab } \\
\text { (hasta } 1 \text { empleado) }\end{array}$ & $\begin{array}{l}\text { 6. Trabajadores } \\
\text { expertos }\end{array}$ & $\begin{array}{l}\text { 9. Obreros } \\
\text { semicalificados }\end{array}$ & 12. Proletariado \\
\hline
\end{tabular}

Fuente: elaboración de los autores, con base en la referencia 1.

a Se refieren al control de la organización del trabajo dentro de la empresa.

b Se refiere a los propietarios de los bienes de producción que tienen como máximo un empleado. 
ted diría que su salud está...", la que fue transformada en una variable dicotómica: buena ( $=0$ [muy bien, bien y más que regular]) o mala (= 1 [regular, menos que regular, mal y muy mal]). Esta pregunta se ha utilizado en diferentes estudios como una medida adecuada de salud global y es una buena predictora de la morbilidad y la mortalidad (20).

Salud mental. El Cuestionario de Salud Global (GHQ-12, por sus siglas en inglés) es un instrumento de cribado que identifica posibles casos de trastorno mental en las personas. La variable dependiente se construyó considerando que los entrevistados que obtienen tres o más puntos — según el GHQ-12- son positivos en el cribado, lo que significa que podrían tener algún trastorno mental (21).

Conductas relacionadas con la salud

Consumo de tabaco. La pregunta fue "En el último mes, ¿Ha fumado al menos un cigarrillo?". Las opciones de respuesta fueron: Sí; No, dejé de fumar hace menos de seis meses; No, dejé de fumar hace más de seis meses; No, nunca he fumado. Solo los que respondieron "sí" se consideraron fumadores.

El consumo de tabaco fue escogido como variable de estudio debido a que existen investigaciones que han encontrado que tal consumo está asociado a la organización del trabajo, los niveles de estrés laboral y las desigualdades socioeconómicas - medidas a partir del nivel de estudios (22). Por otra parte, hay pocos trabajos que analicen la asociación entre clase social y consumo de tabaco, y por ende, muy poca información respecto de por qué estas desigualdades se producen (23).

Práctica de actividad física o deportiva. Se pregunta si el participante, durante el mes anterior a la encuesta, ha practicado deporte o alguna actividad física por 30 minutos o más, y la frecuencia. La variable dicotómica es: "Sí, he realizado actividad una o más veces por semana" (1) y "No, realizo actividad física menos de una vez por semana" o "no he practicado deporte el último mes" $(0)$.

Se incluyó esta variable porque, como han demostrado muchos estudios que analizan estilos de vida o conductas relacionados con la salud, realizar actividad física es una muy buena estrategia para prevenir el estrés, la obesidad, las enfermedades crónicas y las enfermedades del sistema circulatorio (24).

\section{Análisis de datos}

Se emplearon las medidas de CSNM para analizar la estructura de clases de la población chilena, utilizando el número y porcentaje de habitantes en las diferentes posiciones de clase.

Luego se describieron las prevalencias de los diferentes indicadores y conductas relacionados con la salud, y en tercer lugar, se estudió la asociación entre la posición de clase social y los indicadores y conductas relacionados con la salud. Para ello, se ajustaron modelos de regresión multivariada de Poisson robusta estimando la razón de probabilidades (RP) y los intervalos de confianza de 95\% (IC95\%), ajustando por edad y sin ajustar. La categoría de referencia fue el proletario formal, ya que notifica una elevada proporción de participantes y permite identificar desigualdades en relación a las clases sociales más privilegiadas.

Todos los análisis se realizaron por separado para hombres y mujeres. Los datos fueron ponderados según los pesos derivados de una muestra de diseño complejo y analizados con el paquete de programas estadísticos STATA 10.

\section{RESULTADOS}

En el cuadro 1, que muestra la distribución de la población según su clase social, puede verse que una gran proporción de trabajadores en Chile pertenecían a la clase social de obreros semicalificados $(33,3 \%)$, proletario formal $(9,8 \%)$ y trabajadores expertos $(9 \%)$. Las otras categorías que presentaron un porcentaje alto fueron los pequeños burgueses $(18,8 \%)$ y el proletario informal $(6 \%)$. $\mathrm{Al}$ analizar los resultados estratificando por sexo, se pudo observar que una mayor proporción de hombres $(1,6 \%)$ que mujeres $(0,8 \%)$ pertenecían a la clase social de empresarios. La proporción de medianos empresarios también fue más elevada en los hombres (7\%) - frente a $4 \%$ para las mujeres-, mientras que la de proletario informal resultó más alta

CUADRO 1. Distribución de la clase social, según el esquema de Wright, en población abierta de Chile, 2009-2010

\begin{tabular}{|c|c|c|c|c|c|c|}
\hline & \multicolumn{2}{|c|}{ Hombres } & \multicolumn{2}{|c|}{ Mujeres } & \multicolumn{2}{|c|}{ Total } \\
\hline & No. & $\%$ & No. & $\%$ & No. & $\%$ \\
\hline Empresarios & 91 & 1,6 & 30 & 0,8 & 120 & 1,3 \\
\hline Medianos empresarios & 398 & 7,0 & 152 & 4,0 & 551 & 5,8 \\
\hline Pequeños burgueses & 1110 & 19,5 & 681 & 17,9 & 1791 & 18,8 \\
\hline Gerentes expertos & 156 & 2,7 & 109 & 2,9 & 265 & 2,8 \\
\hline Supervisores expertos & 126 & 2,2 & 100 & 2,6 & 226 & 2,4 \\
\hline Trabajadores expertos & 398 & 7,0 & 455 & 11,9 & 853 & 9,0 \\
\hline Gerentes semicalificados & 174 & 3,0 & 132 & 3,5 & 306 & 3,2 \\
\hline Supervisores semicalificados & 348 & 6,1 & 129 & 3,4 & 476 & 5,0 \\
\hline Obreros semicalificados & 1798 & 31,6 & 1368 & 35,9 & 3167 & 33,3 \\
\hline Gerente básicos & 46 & 0,8 & 17 & 0,4 & 63 & 0,7 \\
\hline Supervisores básicos & 38 & 0,7 & 25 & 0,6 & 63 & 0,7 \\
\hline Proletario formal & 678 & 11,9 & 254 & 6,7 & 932 & 9,8 \\
\hline Proletario informal & 256 & 4,5 & 314 & 8,2 & 569 & 6,0 \\
\hline No contestaron & 78 & 1,4 & 42 & 1,1 & 120 & 1,26 \\
\hline
\end{tabular}

Fuente: elaboración de los autores, con base en las referencias 1 y 18. 
en las mujeres $(8,2 \%)$ — contra $4,5 \%$ para los hombres.

La prevalencia de mala salud autopercibida presentó una tasa más alta para los supervisores básicos (51\%), seguidos del proletario informal $(34,2 \%)$, los pequeños burgueses $(33,3 \%)$ y el proletario formal (31,5\%) (cuadro 2). Los medianos empresarios registraron, de forma estadísticamente significativa, una menor percepción de mala salud $(21,6 \%$ [RP 0,68 ; IC95\% 0,46-0,99]) y pudieron observarse algunas diferencias según el sexo. Así, entre los hombres, los que informaron tener una menor percepción de mala salud fueron los medianos empresarios (RP 0,52; IC95\% 0,31-0,87), los gerentes expertos (RP 0,30; IC95\% 0,14-0,66), los supervisores semicalificados (RP 0,60; IC95\% 0,36-0,99) y los obreros semicalificados (RP 0,74; IC95\% 0,55-10). En el grupo de las mujeres, en cambio, notificaron mayor riesgo de tener una mala salud autopercibida las supervisoras básicas (86,9\% [RP 2,38; IC95\% 1,73-3,26]).

Los resultados también muestran que, en general, los empresarios fueron quienes informaron tener una menor prevalencia de problemas de salud mental
$(8,5 \%)$ (cuadro 3). Las tasas más elevadas correspondieron a los supervisores bási$\cos (43,2 \%)$, proletario informal $(28,8 \%)$, supervisores semicalificados $(27,9 \%)$ y proletario formal $(25,0 \%)$. La única diferencia estadísticamente significativa fue que los gerentes básicos registraron una menor prevalencia de riesgo de presentar problemas de salud mental $(11,1 \%$ [RP 0,43; IC95\% 0,21-0,88]). No obstante, sí se observaron diferencias al estratificar los análisis por sexo. En el grupo de hombres, presentaron una menor prevalencia los medianos empresarios (13,9\% [RP 0,59; IC95\% 0,35-0,96]) y los gerentes expertos (10\% [RP 0,43; IC95\% $0,21-0,88])$. Y mientras que entre las mujeres la menor prevalencia fue registrada por las gerentes básicas (6,4\% [RP 0,22; IC95\% 0,07-0,72]), la prevalencia significativamente más alta correspondió a las supervisoras básicas (81\% [RP 2,85; IC95\% 1,82-4,44]).

Los datos expuestos en el cuadro 4 muestran que, al comparar por clases sociales, los empresarios informaron porcentajes más bajos de fumadores (16\% [RP 0,43; IC95\% 0,19-0,99]). Si bien en el grupo de hombres quienes más fu- maban eran los trabajadores expertos y obreros semicalificados - diferencias que dejan de ser significativas al ajustar por edad-, entre las mujeres la mayor prevalencia correspondió a las gerentes semicalificadas (51,4\% [RP 1,91; IC95\% 1,23-2,93]). Es decir que, al contrario de lo verificado con los hombres, las mujeres que fumaban más eran las de clases sociales privilegiadas.

Finalmente, quienes realizaban actividad física o algún deporte con la frecuencia de una o más veces por semana fueron, según los porcentajes registrados, los supervisores expertos (28,5\% [RP 2,45; IC95\% 1,42-4,25 al comparar con los proletarios formales]), los empresarios (28,4\% [RP 2,47; IC95\% 1,21-5,05]), los gerentes expertos (22,8\% [RP 1,94; IC95\% 1,15-3,29]) y los supervisores semicalificados (22,1\% [RP 1,75; IC95\% 1,02-2,98]) (cuadro 5).

\section{DISCUSIÓN}

Luego de haber analizado los indicadores y conductas relacionados con la salud, queda claro que en Chile existen desigualdades en salud asociadas a la

CUADRO 2. Asociación entre la clase social y el estado de salud, según el esquema de Wright y a partir de su razón de probabilidades (RP) e intervalos de confianza de 95\% (IC95\%), en población abierta de Chile, 2009-2010

\begin{tabular}{|c|c|c|c|c|c|c|c|c|c|c|c|c|c|c|c|c|c|c|c|c|c|}
\hline & \multicolumn{7}{|c|}{ Hombres } & \multicolumn{7}{|c|}{ Mujeres } & \multicolumn{7}{|c|}{ Total } \\
\hline & \multirow[b]{2}{*}{$\%$} & \multirow[b]{2}{*}{$\mathrm{RP}^{\mathrm{a}}$} & \multicolumn{2}{|c|}{$\mathrm{IC} 95 \% \mathrm{a}$} & \multirow[b]{2}{*}{$\mathrm{RP}^{\mathrm{b}}$} & \multicolumn{2}{|c|}{$\mathrm{IC} 95 \% \mathrm{~b}$} & \multirow[b]{2}{*}{$\%$} & \multirow[b]{2}{*}{$\mathrm{RP}^{\mathrm{a}}$} & \multicolumn{2}{|c|}{ IC95\%a } & \multirow[b]{2}{*}{$\mathrm{RP}^{\mathrm{b}}$} & \multicolumn{2}{|c|}{ IC95\%b } & \multirow[b]{2}{*}{$\%$} & \multirow[b]{2}{*}{$\mathrm{RP}^{\mathrm{a}}$} & \multicolumn{2}{|c|}{ IC95\%a } & \multirow[b]{2}{*}{$\mathrm{RP}^{\mathrm{b}}$} & \multicolumn{2}{|c|}{ IC95\%b } \\
\hline & & & Mín & Máx & & Mín & Máx & & & Mín & Máx & & Mín & Máx & & & Mín & Máx & & Mín & Máx \\
\hline Empresarios & 16,7 & 0,57 & 0,12 & 2,65 & 0,43 & 0,06 & 3,11 & 7,9 & 0,21 & 0,04 & 1,19 & 0,29 & 0,06 & 1,44 & 14,6 & 0,46 & 0,11 & 0,80 & 0,41 & 0,80 & 2,10 \\
\hline $\begin{array}{l}\text { Medianos } \\
\text { empresarios }\end{array}$ & 15,6 & $0,53^{c}$ & 0,33 & 0,87 & $0,52^{c}$ & 0,31 & 0,87 & 37,3 & 1,00 & 0,62 & 1,60 & 1,01 & 0,62 & 1,63 & 21,6 & $0,69^{c}$ & 0,47 & 0,99 & $0,68^{c}$ & 0,46 & 0,99 \\
\hline $\begin{array}{l}\text { Pequeños } \\
\text { burgueses }\end{array}$ & 30,0 & 1,03 & 0,78 & 1,34 & 0,97 & 0,75 & 1,26 & 38,6 & 1,03 & 0,74 & 1,45 & 1,04 & 0,75 & 1,44 & 33,3 & 1,06 & 0,85 & 1,31 & 1,03 & 0,83 & 1,26 \\
\hline $\begin{array}{l}\text { Gerentes expertos } \\
\text { Supervisores }\end{array}$ & 8,1 & $0,27^{c}$ & 0,12 & 0,59 & $0,30^{c}$ & 0,14 & 0,66 & 29,3 & 0,79 & 0,36 & 1,68 & 0,89 & 0,44 & 1,83 & 16,8 & $0,53^{c}$ & 0,29 & 0,99 & 0,60 & 0,32 & 1,09 \\
\hline expertos & 13,9 & 0,48 & 0,21 & 1,05 & 0,54 & 0,24 & 1,19 & 29,1 & 0,78 & 0,40 & 1,52 & 0,88 & 0,45 & 1,73 & 20,7 & 0,66 & 0,38 & 1,12 & 0,74 & 0,44 & 1,27 \\
\hline $\begin{array}{c}\text { Trabajadores } \\
\text { expertos }\end{array}$ & 15,1 & $0,51^{c}$ & 0,30 & 0,88 & 0,65 & 0,38 & 1,11 & 26,2 & 0,70 & 0,44 & 1,11 & 0,84 & 0,54 & 1,33 & 21,0 & $0,66^{c}$ & 0,47 & 0,95 & 0,82 & 0,56 & 1,16 \\
\hline $\begin{array}{l}\text { Gerentes } \\
\text { semicalificados }\end{array}$ & 19,9 & 0,68 & 0,41 & 1,11 & 0,80 & 0,50 & 1,28 & 30,2 & 0,81 & 0,48 & 1,37 & 0,91 & 0,53 & 1,54 & 24,4 & 0,77 & 0,53 & 1,11 & 0,89 & 0,62 & 1,25 \\
\hline $\begin{array}{l}\text { Supervisores } \\
\text { semicalificados }\end{array}$ & 15,2 & $0,52^{c}$ & 0,31 & 0,88 & $0,60^{c}$ & 0,36 & 0,99 & 25,6 & 0,69 & 0,32 & 1,45 & 0,86 & 0,42 & 1,76 & 18,0 & $0,57^{c}$ & 0,37 & 0,90 & 0,67 & 0,43 & 1,05 \\
\hline $\begin{array}{l}\text { Obreros semi- } \\
\text { calificados }\end{array}$ & 17,8 & 0,61 & 0,31 & 1,18 & $0,74^{\mathrm{c}}$ & 0,55 & 1,00 & 38,0 & 1,02 & 0,73 & 1,43 & 1,21 & 0,85 & 1,70 & 26,5 & 0,84 & 0,66 & 1,06 & 1,01 & 0,79 & 1,28 \\
\hline $\begin{array}{l}\text { Gerentes básicos } \\
\text { Supervisores }\end{array}$ & 18,0 & 0,61 & 0,32 & 1,18 & 0,54 & 0,28 & 1,05 & 28,7 & 0,77 & 0,27 & 2,16 & 0,74 & 0,27 & 1,99 & 20,8 & 0,66 & 0,37 & 1,16 & 0,61 & 0,35 & 1,05 \\
\hline básicos & 27,4 & 0,94 & 0,37 & 2,34 & 0,93 & 0,32 & 2,64 & 86,9 & $2,33^{c}$ & 1,69 & 3,21 & $2,38^{c}$ & 1,73 & 3,26 & 51,0 & 1,62 & 0,95 & 2,77 & 1,62 & 0,90 & 1,46 \\
\hline Proletario formal & 29,3 & 1,00 & $\ldots$ & $\ldots$ & 1,00 & $\ldots$ & $\ldots$ & 37,3 & 1,00 & $\ldots$ & $\ldots$ & 1,00 & $\ldots$ & $\ldots$ & 31,5 & 1,00 & $\ldots$ & $\ldots$ & $\ldots$ & $\ldots$ & $\ldots$ \\
\hline Proletario informal & 30,9 & 1,06 & 0,76 & 1,47 & 1,10 & 0,79 & 1,51 & 36,9 & 0,99 & 0,68 & 1,43 & 1,04 & 0,72 & 1,50 & 34,2 & 1,09 & 0,85 & 1,40 & 1,14 & 0,51 & 1,92 \\
\hline No contestaron & 9,3 & $0,32^{c}$ & 0,10 & 0,98 & 0,39 & 0,12 & 1,03 & 58,6 & 1,57 & 0,92 & 2,69 & $1,77^{c}$ & 1,07 & 2,91 & 26,7 & 0,85 & 0,43 & 1,67 & 0,99 & 1,01 & 1,03 \\
\hline
\end{tabular}


CUADRO 3. Asociación entre la clase social y el riesgo de presentar un problema de salud mental, según el esquema de Wright y a partir de su razón de probabilidades (RP) e intervalos de confianza de 95\% (IC95\%), en población abierta de Chile, 2009-2010

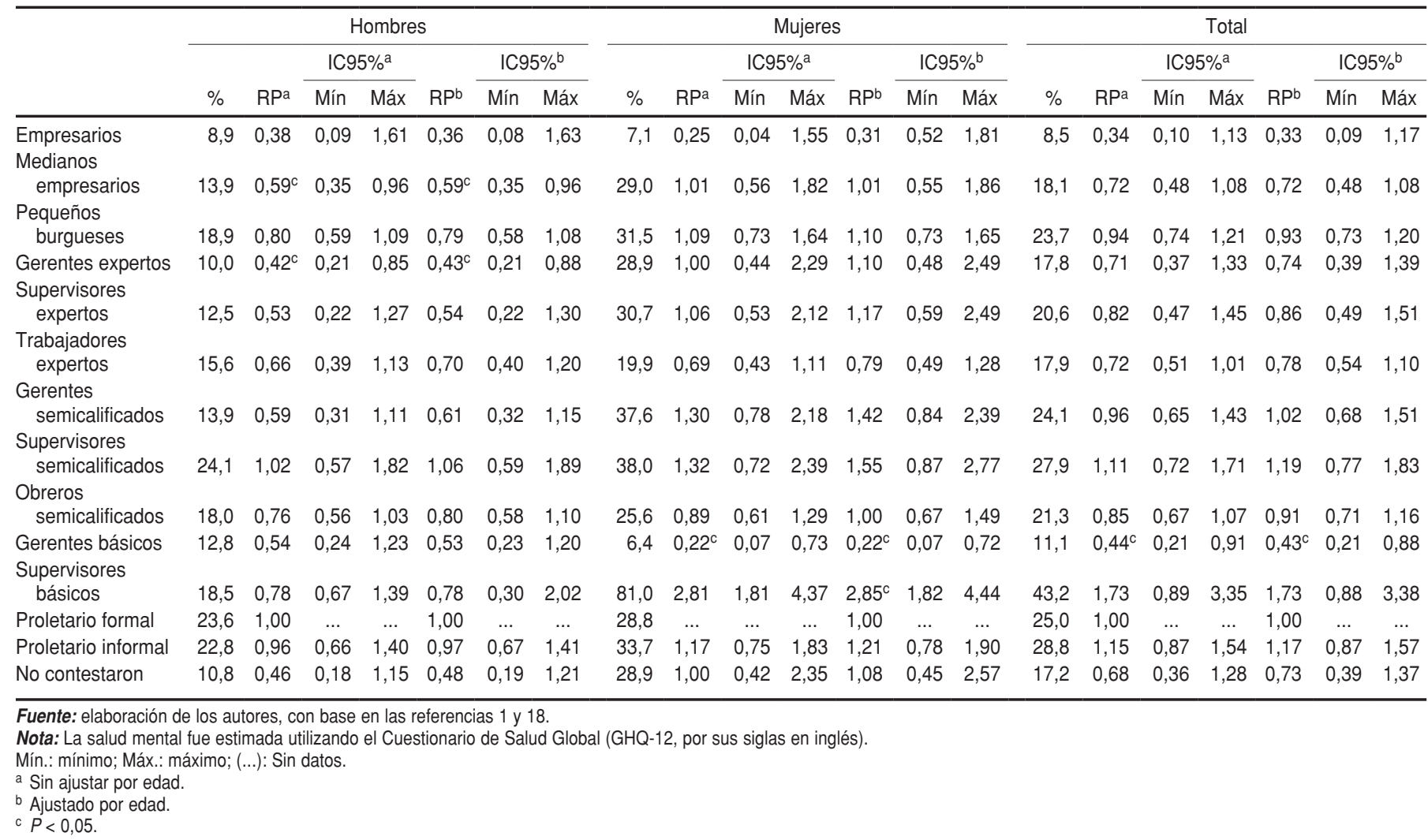

CUADRO 4. Asociación entre la clase social y la prevalencia de fumadores, según el esquema de Wright y a partir de su razón de probabilidades (RP) e intervalos de confianza de 95\% (IC95\%), en población abierta de Chile, 2009-2010

\begin{tabular}{|c|c|c|c|c|c|c|c|c|c|c|c|c|c|c|c|c|c|c|c|c|c|}
\hline & \multicolumn{7}{|c|}{ Hombres } & \multicolumn{7}{|c|}{ Mujeres } & \multicolumn{7}{|c|}{ Total } \\
\hline & \multirow[b]{2}{*}{$\%$} & \multirow[b]{2}{*}{$\mathrm{RP}^{\mathrm{a}}$} & \multicolumn{2}{|c|}{ IC95\% ${ }^{a}$} & \multirow[b]{2}{*}{$\mathrm{RP}^{\mathrm{b}}$} & \multicolumn{2}{|c|}{ IC95\% ${ }^{\mathrm{b}}$} & \multirow[b]{2}{*}{$\%$} & \multirow[b]{2}{*}{$\mathrm{RP}^{\mathrm{a}}$} & \multicolumn{2}{|c|}{$1 \mathrm{C} 95 \%{ }^{\mathrm{a}}$} & \multirow[b]{2}{*}{$\mathrm{RP}^{\mathrm{b}}$} & \multicolumn{2}{|c|}{ IC95\% ${ }^{\mathrm{b}}$} & \multirow[b]{2}{*}{$\%$} & \multirow[b]{2}{*}{$\mathrm{RP}^{\mathrm{a}}$} & \multicolumn{2}{|c|}{ IC95\% ${ }^{a}$} & \multirow[b]{2}{*}{$\mathrm{RP}^{\mathrm{b}}$} & \multicolumn{2}{|c|}{ IC95\% ${ }^{\mathrm{b}}$} \\
\hline & & & Mín & Máx & & Mín & Máx & & & Mín & Máx & & Mín & Máx & & & Mín & Máx & & Mín & Máx \\
\hline $\begin{array}{l}\text { Medianos } \\
\text { empresarios }\end{array}$ & 27,3 & 0,71 & 0,48 & 1,04 & 0,70 & 0,48 & 1,02 & 24,8 & 1,04 & 0,59 & 1,82 & 1,02 & 0,58 & 1,79 & 26,6 & 0,77 & 0,56 & 1,06 & 0,76 & 0,55 & 1,04 \\
\hline $\begin{array}{l}\text { Pequeños } \\
\text { burgueses }\end{array}$ & 37,8 & 0,98 & 0,78 & 1,23 & 1,01 & 0,82 & 1,25 & 29,1 & 1,22 & 0,81 & 1,83 & 1,19 & 0,80 & 1,78 & 34,5 & 1,00 & 0,81 & 1,22 & 1,01 & 0,83 & 1,22 \\
\hline $\begin{array}{l}\text { Trabajadores } \\
\text { expertos }\end{array}$ & 53,1 & $1,38^{c}$ & 1,06 & 1,77 & 1,12 & 0,87 & 1,44 & 35,3 & 1,48 & 0,97 & 2,24 & 1,22 & 0,81 & 1,83 & 43,6 & $1,26^{c}$ & 1,01 & 1,58 & 1,03 & 0,83 & 1,28 \\
\hline $\begin{array}{l}\text { Gerentes } \\
\text { semicalificados }\end{array}$ & 48,3 & 1,25 & 0,91 & 1,72 & 1,07 & 0,80 & 1,44 & 51,4 & $2,15^{c}$ & 1,39 & 3,13 & $1,91^{\mathrm{C}}$ & 1,23 & 2,93 & 49,6 & $1,43^{c}$ & 1,11 & 1,84 & 1,25 & 0,98 & 1,60 \\
\hline $\begin{array}{l}\text { Supervisores } \\
\text { semicalificados }\end{array}$ & 43,9 & 1,14 & 0,80 & 1,62 & 1,00 & 0,68 & 1,47 & 25,2 & 1,05 & 0,59 & 1,89 & 0,84 & 0,47 & 1,51 & 38,9 & 1,12 & 0,82 & 1,54 & 0,96 & 0,68 & 1,34 \\
\hline básicos & 36,5 & 0,94 & 0,47 & 1,90 & 0,94 & 0,51 & 1,73 & 15,5 & 0,65 & 0,15 & 2,84 & 0,64 & 0,15 & 2,79 & 28,2 & 0,81 & 0,40 & 1,66 & 0,81 & 0,41 & 1,59 \\
\hline Proletario formal & 38,6 & 1,00 & $\ldots$ & $\ldots$ & 1,00 & $\ldots$ & $\ldots$ & 23,9 & 1,00 & $\ldots$ & $\ldots$ & 1,00 & $\ldots$ & $\ldots$ & 34,6 & 1,00 & $\ldots$ & $\ldots$ & 1,00 & $\ldots$ & $\ldots$ \\
\hline Proletario informal & 40,5 & 1,05 & 0,79 & 1,38 & 0,99 & 0,75 & 1,31 & 28,0 & 1,17 & 0,71 & 1,92 & 1,11 & 0,67 & 1,80 & 33,6 & 0,97 & 0,75 & 1,26 & 0,92 & 0,71 & 1,18 \\
\hline No contestaron & 50,9 & 1,32 & 0,80 & 2,17 & 1,07 & 0,62 & 1,84 & 41,0 & 1,72 & 0,82 & 3,58 & 1,55 & 0,75 & 3,19 & 47,4 & 1,37 & 0,89 & 2,11 & 1,16 & 0,74 & 1,80 \\
\hline
\end{tabular}


CUADRO 5. Asociación entre la clase social y la práctica de actividad física durante el mes anterior a la encuesta, según el esquema de Wright y a partir de su razón de probabilidades (RP) e intervalos de confianza de 95\% (IC95\%), en población abierta de Chile, 2009-2010

\begin{tabular}{|c|c|c|c|c|c|c|c|c|c|c|c|c|c|c|c|c|c|c|c|c|c|}
\hline & \multicolumn{7}{|c|}{ Hombres } & \multicolumn{7}{|c|}{ Mujeres } & \multicolumn{7}{|c|}{ Total } \\
\hline & \multirow[b]{2}{*}{$\%$} & \multirow[b]{2}{*}{$\mathrm{RP}^{\mathrm{a}}$} & \multicolumn{2}{|c|}{ IC95\%a } & \multirow[b]{2}{*}{$\mathrm{RP}^{\mathrm{b}}$} & \multicolumn{2}{|c|}{ IC95\%b } & \multirow[b]{2}{*}{$\%$} & \multirow[b]{2}{*}{$\mathrm{RP}^{\mathrm{a}}$} & \multicolumn{2}{|c|}{ IC95\%a } & \multirow[b]{2}{*}{$\mathrm{RP}^{\mathrm{b}}$} & \multicolumn{2}{|c|}{ IC95\%b } & \multirow[b]{2}{*}{$\%$} & \multirow[b]{2}{*}{$\mathrm{RP}^{\mathrm{a}}$} & \multicolumn{2}{|c|}{ IC95\%a } & \multirow[b]{2}{*}{$\mathrm{RP}^{\mathrm{b}}$} & \multicolumn{2}{|c|}{ IC95\%b } \\
\hline & & & Mín & Máx & & Mín & Máx & & & Mín & Máx & & Mín & Máx & & & Mín & Máx & & Mín & Máx \\
\hline Empresarios & 6,6 & $3,56^{c}$ & 1,46 & 8,71 & $3,41^{c}$ & 2,05 & 5,68 & 3,1 & 0,43 & 0,07 & 2,72 & 0,32 & 0,05 & 2,15 & 28,4 & $3,00^{c}$ & 1,26 & 7,15 & $2,47^{c}$ & 1,21 & 5,05 \\
\hline $\begin{array}{l}\text { Medianos } \\
\text { empresarios }\end{array}$ & 16,8 & 1,63 & 0,81 & 3,29 & 1,57 & 0,84 & 2,94 & 14,5 & 1,99 & 0,69 & 5,71 & 1,95 & 0,70 & 1,46 & 16,1 & 1,71 & 0,95 & 3,07 & 1,65 & 0,96 & 2,82 \\
\hline $\begin{array}{l}\text { Pequeños } \\
\text { burgueses }\end{array}$ & 14,1 & 1,37 & 0,82 & 2,28 & 1,45 & 0,90 & 2,32 & 10,7 & 1,46 & 0,66 & 3,40 & 1,44 & 0,62 & 3,35 & 12,8 & 1,35 & 0,88 & 2,07 & 1,37 & 0,91 & 2,07 \\
\hline $\begin{array}{l}\text { Gerentes expertos } \\
\text { Supervisores }\end{array}$ & 28,1 & $2,73^{c}$ & 1,56 & 4,78 & $2,24^{c}$ & 1,29 & 3,89 & 15,2 & 2,08 & 0,70 & 6,19 & 1,82 & 0,58 & 5,76 & 22,8 & $2,40^{c}$ & 1,45 & 3,99 & $1,94^{c}$ & 1,15 & 3,29 \\
\hline $\begin{array}{c}\text { expertos } \\
\text { Trabajadores }\end{array}$ & 28,2 & $2,75^{c}$ & 1,35 & 5,58 & $2,25^{c}$ & 1,12 & 4,53 & 28,8 & $3,96^{c}$ & 1,53 & 10,20 & $3,50^{c}$ & 1,32 & 9,23 & 28,5 & $3,01^{c}$ & 1,75 & 5,17 & $2,45^{c}$ & 1,42 & 4,25 \\
\hline $\begin{array}{l}\text { expertos } \\
\text { Gerentes }\end{array}$ & 24,1 & $2,34^{c}$ & 1,41 & 3,90 & 1,56 & 0,96 & 2,54 & 15,6 & 2,13 & 0,93 & 4,91 & 1,78 & 0,77 & 4,09 & 19,5 & $2,07^{c}$ & 1,36 & 3,15 & 1,43 & 0,94 & 2,17 \\
\hline $\begin{array}{l}\text { semicalificados } \\
\text { Supervisores }\end{array}$ & 29,8 & $2,90^{\mathrm{C}}$ & 1,66 & 5,04 & $2,14^{c}$ & 1,26 & 3,63 & 4,3 & 0,60 & 0,20 & 1,70 & 0,53 & 0,18 & 1,53 & 18,8 & $1,99^{c}$ & 1,16 & 3,30 & 1,55 & 0,92 & 2,61 \\
\hline $\begin{array}{l}\text { semicalificados } \\
\text { Obreros }\end{array}$ & 25,4 & $2,47^{c}$ & 1,35 & 4,50 & $1,91^{c}$ & 1,06 & 3,44 & 13,2 & 1,81 & 0,65 & 5,02 & 1,46 & 0,52 & 4,11 & 22,1 & $2,33^{c}$ & 1,36 & 3,99 & $1,75^{\mathrm{c}}$ & 1,02 & 2,98 \\
\hline semicalificados & 22,4 & $2,18^{c}$ & 1,47 & 3,22 & 1,46 & 0,96 & 2,24 & 8,4 & 1,16 & 0,52 & 2,58 & 0,98 & 0,43 & 2,26 & 16,3 & $1,73^{c}$ & 1,21 & 2,46 & 1,23 & 0,84 & 1,80 \\
\hline $\begin{array}{l}\text { Gerentes } \\
\text { básicos }\end{array}$ & 20,8 & 2,02 & 0,81 & 5,05 & $2,57^{c}$ & 1,09 & 6,07 & 5,0 & 0,68 & 0,14 & 3,25 & 0,71 & 0,15 & 3,39 & 16,6 & 1,75 & 0,72 & 4,23 & 2,07 & 0,89 & 4,86 \\
\hline $\begin{array}{l}\text { Supervisores } \\
\text { básicos }\end{array}$ & 7,0 & 0,68 & 0,21 & 2,27 & 0,66 & 0,20 & 2,14 & 59,3 & $8,14^{c}$ & 2,86 & 23,20 & $8,09^{c}$ & 2,79 & 23,40 & 27,7 & 2,93 & 0,92 & 9,26 & 2,90 & 0,88 & 9,50 \\
\hline Proletario formal & 10,3 & 1,00 & $\ldots$ & $\ldots$ & 1,00 & $\ldots$ & $\ldots$ & 7,3 & 1,00 & $\ldots$ & $\ldots$ & 1,00 & $\ldots$ & $\ldots$ & 9,5 & 1,00 & $\ldots$ & $\ldots$ & 1,00 & $\ldots$ & $\ldots$ \\
\hline Proletario informal & 16,4 & 1,59 & 0,96 & 2,65 & 1,39 & 0,86 & 2,22 & 9,1 & 1,25 & 0,47 & 3,30 & 1,18 & 0,45 & 3,09 & 12,4 & 1,31 & 0,82 & 2,09 & 1,17 & 0,74 & 1,84 \\
\hline No contestaron & 36,6 & $3,56^{c}$ & 1,65 & 7,69 & $2,30^{c}$ & 1,09 & 4,82 & 2,6 & 0,35 & 0,86 & 1,47 & 0,32 & 0,08 & 1,33 & 24,6 & $2,60^{c}$ & 1,15 & 5,89 & 1,90 & 0,88 & 4,07 \\
\hline
\end{tabular}

Fuente: elaboración de los autores, con base en las referencias 1 y 18.

Mín.: Mínimo; Máx.: Máximo; (...): Sin datos

a Sin ajustar por edad.

b Ajustado por edad.

c $P<0,05$.

clase social. La mayor prevalencia de problemas de salud corresponde a los supervisores básicos y semicalificados, y al proletario informal. Asimismo, tienen significativamente mejores indicadores de salud los empresarios y los gerentes expertos, seguidos de los trabajadores expertos y de los obreros semicalificados. Estos resultados guardan consistencia interna, entre los diferentes indicadores y conductas de salud, así como externa, en concordancia con los resultados de estudios previos (3, 6-8). También son notables las diferencias de género en relación a la clase social, observándose entre los hombres de clase social más privilegiada un patrón de mejores indicadores y conductas de salud, lo que no sucede del mismo modo con las mujeres. Por ejemplo, mientras que las que más fuman corresponden al grupo de gerentes, sus pares (gerentes) hombres están entre los que fuman menos.

\section{Estructura de clase}

La clase de empresarios representa a $1,3 \%$ de la población, de modo similar a lo verificado en estudios realizados en Chile (1,5\%) (19), Barcelona (1,4\%) (3), Suecia $(0,7 \%)(1)$ y Noruega $(0,8 \%)(1), y$ por debajo del $2 \%$ descrito por Wright en 2000 para países como Canadá, Estados Unidos, Japón y el Reino Unido (1). De ello se puede inferir que el proceso de globalización desarrollado en el siglo pasado ha impuesto una estructura de clases parecida en países ricos y de ingresos medios que adhieren a la economía de mercado (1).

El porcentaje - en la muestra- de medianos empresarios $(5,8 \%)$ también concuerda con los obtenidos en diversos trabajos en Barcelona $(6,6 \%)$, Estados Unidos $(6 \%)$ y Japón $(6,2 \%)(1,3)$. En este tipo de empresas medianas es muy difícil distinguir su grado de fomalidad/informalidad, lo que es un dato importante debido a la gran cantidad de trabajo informal en América Latina (19) y pone en evidencia las limitaciones de los modelos anglosajones para captar la realidad de otros países en dicha región (14).

En Chile, los pequeños burgueses corresponderían a $20 \%$ de la población, resultado que se encuentra por encima del $10,7 \%$ registrado en investigaciones previas en el país, las cuales refieren una elevada heterogeneidad interna en esta categoría (19). En este sentido, el criterio utilizado fue la propiedad, por lo que probablemente se hayan incluido también profesionales liberales y otros profesionales que guardan más cercanía con el proletario informal que con los pequeños burgueses, tanto por sus condiciones de trabajo más precarias como por su inserción al mercado laboral principalmente asociada a la necesidad de subsistencia. El trabajo efectuado por Wright —en seis países- concluyó que los porcentajes referidos a los pequeños burgueses son los que presentan una mayor variación, oscilando entre $5 \%$ en Suecia y $23 \%$ en Japón (1).

La suma del proletario formal, el proletario informal y los trabajadores semicalificados representa a $49,1 \%$ de la población chilena, proporción similar a las verificadas en estudios realizados en otros países, donde los puestos de trabajo de clase trabajadora no gerencial corresponden a alrededor de $60 \%$ de la población $(1,3)$. Más aún, estos resulta- 
dos desmienten la afirmación de que se esté llegando a una "sociedad del conocimiento" en los países desarrollados, en la que la mayoría de los trabajos requiere un alto nivel de especialización técnica (25). La frecuente recomendación de la literatura anglosajona sobre la necesidad de incrementar la educación de la población (26) se enfrenta así con un hecho que la pone en pugna: la mayor parte de los trabajos -en países ricos y de ingresos medios- no necesita de un alto grado de especialización (27).

$\mathrm{Al}$ igual que ocurre en países más desarrollados $(3,28)$, en Chile existe un porcentaje mayor de empresarios y medianos empresarios hombres que mujeres, lo que es una muestra más de las desigualdades de género que padecen esos países.

\section{Clase social y su asociación con salud y conductas relacionadas con la salud}

Como ya se dijo, existe una vinculación entre el estado de salud autopercibido y las medidas de CSNM. En general, los medianos empresarios, los gerentes expertos y los trabajadores expertos presentan una mejor salud, en sintonía con lo observado en estudios anteriores $(3,6)$. También pueden verse claras desigualdades de clase social asociadas al consumo de tabaco. En efecto, el grupo de empresarios fuma significativamente menos que el proletario formal, lo que puede deberse a una mayor exposición a situaciones de estrés laboral que sus superiores en la jerarquía empresarial (11). Otro aspecto que vale la pena mencionar es que las clases trabajadoras tienen menos acceso a conductas saludables - por ejemplo, practicar algún deporte-, puesto que este tipo de actividades requiere de más tiempo libre y de más recursos para acceder a los equipamientos deportivos, o contar al menos con espacios adecuados y cercanos al hogar (29).

Las desigualdades de género y clase asociadas al consumo de tabaco vienen dadas por la mayor prevalencia de consumo en mujeres de clases sociales privilegiadas y en las que tienen más autoridad, como las supervisoras y gerentes. Por su parte, los hombres que fuman más son los trabajadores expertos y obreros semicalificados. Esta tendencia - la mayor presencia de fumadoras entre mujeres de clase social privilegiadafue observada igualmente en España en los ochenta, revirtiéndose en la década siguiente que mostró una mayor prevalencia en las clases menos privilegiadas (14). De hecho, ha sido documentado que la epidemia del tabaquismo empieza primero en los hombres y las personas de clases privilegiadas, para después pasar a las mujeres y las personas de clases trabajadoras (11). Con el ingreso relativamente reciente de las mujeres al mercado laboral, y su conquista de mayores libertades y espacios - lo que con frecuencia ocurre antes en las clases privilegiadas-, se ha observado que inicialmente suelen adoptar comportamientos de riesgo que son más comunes en los hombres.

En relación al ejercicio y la actividad física, los estudios difieren en sus resultados: algunos registran una menor actividad en personas de clase social menos privilegiada (12), y otros una prevalencia mayor de inactividad entre las de clases sociales más privilegiadas $(13,14)$. Los resultados del presente estudio informan una mayor actividad física entre la clase social más privilegiada y entre los hombres.

Los valores obtenidos sobre los supervisores básicos, para hombres y mujeres, y los de supervisoras semicalificadas, para las mujeres, refuerzan la presencia de una posición contradictoria de clase (1), que ya ha sido descrita empíricamente en investigaciones desarrolladas en España (3, 6-8) y Estados Unidos (5). Este tipo de información permite concluir que muchas desigualdades en salud no pueden ser explicadas mediante los enfoques convencionales basados en la gradación - como nivel de ingresos, educación y ocupación-, sino que requieren perspectivas distintas que hagan hincapié en los componentes sociales que originan tales disparidades (5).

En la misma línea, el hecho de que los empresarios y los gerentes expertos presenten mejores indicadores y conductas relacionados con la salud favorece la idea de que las relaciones de producción están basadas en el mecanismo de dominación, donde "empleadores" $\mathrm{y}$ "empleados" forman un todo interdependiente signado por una relación antagónica.

El análisis realizado encontró también que hay menos mujeres que hombres que ocupen posiciones jerárquicas altas, como empresarias y medianas empresarias, y que entre las mujeres que las ocupan, no se observan ventajas en términos de salud o conductas relacionadas con la salud. Esto puede deberse al efecto de patriarcado o discriminación de género sobre la salud de las mujeres.

\section{Limitaciones}

Al tratarse de un estudio transversal, el presente trabajo tiene las limitaciones propias de este tipo de enfoque. Por esa razón, será recomendable complementar estos resultados con el desarrollo de futuros estudios de tipo longitudinal, los cuales posibilitarán poner el foco en el impacto en el tiempo de la clase social sobre la salud de la población trabajadora. Por otro lado, al ajustar los modelos, se acaban comparando todas las clases sociales con los proletarios formales, lo que dificulta el resto de comparaciones entre clases.

\section{Conclusiones y recomendaciones}

En Chile, existen desigualdades en salud asociadas a la clase social de la población. Las personas pertenecientes a las clases sociales más privilegiadas, como los empresarios y los gerentes expertos, presentan un mejor estado de salud autopercibido, menos comportamientos perjudiciales para la salud $y$ más comportamientos saludables. Por el contrario, la población de clase trabajadora - trabajadores formales, pero principalmente los informales-y los supervisores básicos refieren tener una peor salud y estilos de vida menos saludables.

Hay que tener en cuenta también que ese patrón de mejor salud entre las clases sociales más privilegiadas se ve con mayor claridad en los hombres que en las mujeres, lo que puede estar vinculado a las dificultades que afrontan las mujeres al ocupar posiciones con un mayor nivel de poder, en contextos donde existen desigualdades de género. En ese sentido, sería bueno propiciar un debate sobre las posibilidades reales de éxito de las intervenciones dirigidas a mejorar las conductas relacionadas con la salud que no toman en consideración el contexto social, de trabajo - y de vida - en que las personas están insertas. El análisis de tales conductas como "opción" o "elección" individual pierde gran parte de su significado si no se encuentra debidamente contextualizado. Por esa razón, las medidas de CSNM resultan cruciales a la hora de entender cómo las relaciones de poder que estructuran la sociedad a 
través del trabajo determinan un mayor o menor acceso a la salud y a estilos de vida saludables.

Los resultados del presente estudio resaltan la importancia de los diferentes recursos y oportunidades ligados a la clase social, como factores determinantes de un mayor acceso a la salud, tanto física como mental. En concordancia con lo anterior, la Primera Encuesta Nacional de Condiciones de Empleo, Trabajo, Salud y Calidad de Vida de los Trabajado- res y Trabajadoras en Chile ha provisto datos sumamente valiosos, que demuestran que las conductas relacionadas con la salud no son distribuidas de manera igualitaria entre las distintas clases sociales. Por todo lo expuesto, se recomienda incrementar las políticas sociales destinadas a lograr una mayor igualdad de género en el país. Asimismo, y a la luz de la información examinada sobre el creciente número de trabajadores informales, resulta perentorio realizar nuevas investigaciones que analicen críticamente los efectos de las políticas de flexibilización del mercado laboral en términos de salud pública.

Financiamiento. El presente estudio fue financiado por el Ministerio de Salud de Chile, en el marco de la agenda de equidad en salud 2008-2010.

Conflictos de interés. Ninguno declarado por los autores.

\section{REFERENCIAS}

1. Wright EO. Class counts: Comparative studies in class analysis. Cambridge: Cambridge University Press; 2000.

2. Wright EO. Classes. London: Verso; 1985.

3. Borrell C, Muntaner C, Benach J, Artazcoz L. Social class and self-reported health status among men and women: What is the role of work organisation, household material standards and household labour? Soc Sci Med. 2004;58(10):1869-87.

4. Muntaner C, Parsons P. Income, social stratification, class and private health insurance: A study of the Baltimore metropolitan area. Int J Health Serv. 1996;26: 655-71.

5. Muntaner C, Eaton W, Diala C, Kessler R, Sorlie P. Social class, assets, organizational control and the prevalence of common groups of psychiatric disorders. Soc Sci Med. 1998;47:2043-53.

6. Muntaner C, Borrell C, Benach J, Pasarín MI, Fernandez E. The associations of social class and social stratification with patterns of general and mental health in a Spanish population. Int J Epidemiol. 2003;32:950-8.

7. Ortiz-Hernández L, López-Moreno S, Borges G. Desigualdad socioeconómica y salud mental: Revisión de la literatura latinoamericana. Cad Saude Publica. 2007;23(6):1255-72.

8. Muntaner C, Borrell C, Solá J, Marí-Dell'Olmo M, Chung H, Rodríguez-Sanz M, et al. Capitalists, managers, professionals and mortality: Findings from the Barcelona social class and all cause mortality longitudinal study. Scand J Public Health. 2009;37(8):826-38.

9. Wohlfarth T. Socioeconomic inequality and psychopathology: Are socioeconomic status and social class interchangeable? Soc Sci Med. 1997;45(3):399-410.

10. Galobardes B, Shaw M, Lawlor DA, Lynch JW, Davey SG. Indicators of socioeconomic position (part 2). J Epidemiol Community Health. 2006;60:95-101.

11. Thun M, Peto R, Boreham J, Lopez AD. Stages of the cigarette epidemic on entering its second century. Tob Control. 2012;21(2):96-101.
12. Makinen $\mathrm{T}$, Borodulin $\mathrm{K}$, Laatikainen $\mathrm{T}$, Fogelholm M, Prattala R. Twenty-five year socioeconomic trends in leisure time and commuting physical activity among employed Finns. Scand J Med Sci Sports. 2009;19(2): 188-97.

13. Stalsberg R, Pedersen AV. Effects of socioeconomic status on the physical activity in adolescents: A systematic review of the evidence. Scand J Med Sci Sports. 2010;20(3):368-83.

14. Borrell C, Rueb M, Pasarin MI, Rohlfs I, Ferrando J, Fernández E. Trends in Social Class Inequalities in Health Status, HealthRelated Behaviors and Health Services Utilization in a Southern European Urban Area (1983-1994). Prev Med. 2000;31(6):691-701.

15. Muntaner C, Rocha KB, Borrell C, Vallebuona C, Ibáñez C, Benach J, et al. Social Class and health in Latin America. Rev Panam Salud Publica. 2012;31(2):166-75.

16. Muntaner C, Borrell C, Vanroelen C, Chung $\mathrm{H}$, Benach J, Kim IH, et al. Employment relations, social class and health: A review and analysis of conceptual and measurement alternatives. Soc Sci Med. 2010;71(12):2130-40.

17. Borrell C, Artazcoz L. Las desigualdades de género en salud: Retos para el futuro. Rev Esp Salud Publica. 2008;82:245-9.

18. Chile, Ministerio de Salud. Primera encuesta nacional de empleo, trabajo, salud y calidad de vida de los trabajadores y trabajadoras en Chile. Informe interinstitucional, 2011. Disponible en: http://www.saludytrabajo. cl/wp-content/uploads/2011/09/INTE RINSTITUCIONAL_BAJA.pdf Acceso el 10 de abril de 2013.

19. Portes A, Hoffman K. La estructura de clase en América Latina: Composición y cambios durante la era neoliberal. En: Franco R, Leon A, Atria R, eds. Estratificación y movilidad social en América Latina. Santiago de Chile: CEPAL; 2007. Pp. 559-604.

20. Idler E, Benyamini Y. Self-Rated Health and Mortality: A Review of Twenty-Seven Community Studies. J Health Soc Beha. 1997;38(1):21-37.
21. McDowell I. Measuring Health: A guide to rating scales and questionnaires. $3{ }^{\text {a }}$ ed. New York: Oxford; 2006.

22. Albertsen K, Borg V. A systematic review of the impact of work environment on smoking cessation, relapse and amount smoked. Prev Med. 2006;43:291-305.

23. Jha P, Peto R, Zatonski W, Boreham J, Jarvis $\mathrm{MJ}$, Lopez $\mathrm{AD}$. Social inequalities in male mortality and in male mortality from smoking: Indirect estimation from national death rates in England and Wales, Poland and North America. Lancet. 2006;368(9533): 367-70.

24. Yen IH, Kaplan GA. Poverty area residence and changes in physical activity level: Evidence from the Alameda County Study. Am J Public Health. 1998;88(11):1709-12.

25. UNESCO. Towards Knowledge Societies. UNESCO World Report. París: UNESCO; 2005.

26. Brown DC, Hayward MD, Montez JK, Hummer RA, Chiu CT, Hidajat MM. The Significance of Education for Mortality Compression in the United States. Demography. 2012;49(3):819-40.

27. Muntaner C, Benach J, Tarafa G, Chung J. The welfare state and global health: Latin America, the Arab world and the politics of social class. Gac Sanit. 2011;25(6):445-7.

28. Wright EO, Baxter J, Birkelund E. The Gender Gap in Workplace Authority: A Cross-National Study. Am Sociol Rev. 1995;60(3):407-35.

29. Ross CE. Walking, exercising, and smoking: Does neighborhood matter? Soc Sci Med. 2000;51(2):265-74.

Manuscrito recibido el 3 de julio de 2012. Aceptado para publicación, tras revisión, el 17 de diciembre de 2012. 
ABSTRACT Objective. To analyze links between social class and health-related indicators and behaviors in Chilean workers, from a neo-Marxian perspective.

Social class, health inequalities, and health-related behaviors of working people in Chile

Key words
Methods. A cross-sectional study based on the First National Survey on Employment, Work, Health, and Quality of Life of Workers in Chile, done in 2009-2010 ( $n=9503)$. Dependent variables were self-perceived health status and mental health, examined using the General Health Questionnaire (GHQ-12). Health-related behavior variables included tobacco use and physical activity. The independent variable was neoMarxian social class. Descriptive analyses of prevalence were performed and odds ratio (OR) models and 95\% confidence intervals (95\% CI) were estimated.

Results. Medium employers (between 2 and 10 employees) reported a lower prevalence of poor health ( $21.6 \%$ [OR $0.68 ; 95 \%$ CI $0.46-0.99])$. Unskilled managers had the lowest mental health risk (OR 0.43; 95\%CI 0.21-0.88), with differences between men and women. Large employers (more than 10 employees) reported smoking the least, while large employers, expert supervisors, and semi-skilled workers engaged in significantly more physical activity.

Conclusions. Large employers and expert managers have the best health-related indicators and behaviors. Formal proletarians, informal proletarians, and unskilled supervisors, however, have the worst general health indicators, confirming that social class is a key determinant in the generation of population health inequalities.

Social class; health inequalities; smoking; motor activity; gender and health; health behavior; Chile. 